\title{
O tratamento holístico como alternativa pelo enfermeiro no controle da dor oncológica: relato de experiência
}

\author{
Holistic treatment as an alternative by nurses in oncologic pain control: experience report \\ El tratamiento holístico como alternativa por parte de enfermeras en el control del dolor \\ oncológico: informe de experiencia
}

Recebido: 17/11/2021 | Revisado: 27/11/2021 | Aceito: 27/11/2021 | Publicado: 09/12/2021

Kézia Dias Lopes

ORCID: https://orcid.org/0000-0002-5302-9945

Centro Universitário Metropolitano da Amazônia, Brasil E-mail: keziadilopes@gmail.com.br

Miriam Souza Oliveira

ORCID: https://orcid.org/0000-0002-0561-631X

Centro Universitário Metropolitano da Amazônia, Brasil

E-mail:miriamsouzaoliveira123@gmail.com.br

Lucimara Ribeiro da Silva

ORCID: https://orcid.org/0000-0002-7712-4963

Centro Universitário Metropolitano da Amazônia, Brasil

E-mail: luci.silva702@hotmail.com

Tayná de Paula Furtado de Oliveira

ORCID: https://orcid.org/0000-0003-3207-0938

Centro Universitário Metropolitano da Amazônia, Brasil

E-mail: tayfurtado9@gmail.com.br

Mayara Pinheiro Mendes

ORCID: https://orcid.org/0000-0002-9830-3426 Centro Universitário Metropolitano da Amazônia, Brasil

E-mail: mayarabloom17@gmail.com.br

Thamires Cabral Freitas

ORCID: https://orcid.org/0000-0003-4825-9428 Centro Universitário Metropolitano da Amazônia, Brasil

E-mail:thamicabra102081999@gmail.com.br

Isabely da Silva Mota

ORCID: https://orcid.org/0000-0002-3579-6526 Centro Universitário Metropolitano da Amazônia, Brasil E-mail:isabelymota92@gmail.com.br

Amanda Rodrigues Figueiredo

ORCID: https://orcid.org/0000-0001-5719-9143 Centro Universitário Metropolitano da Amazônia, Brasil E-mail:amandar.figueiredo29@gmail.com.br

David Alfredo Cordeiro da Silva ORCID: https://orcid.org/0000-0001-8462-7430 Centro Universitário Metropolitano da Amazônia, Brasil E-mail:davidalfredosilvaalfre@gmail.com.br

Milena Farah Damous Castanho Ferreira

ORCID: https://orcid.org/0000-0002-0645-2046

Centro Universitário Metropolitano da Amazônia, Brasil

E-mail:milenaferreira@famaz.edu.br

\section{Resumo}

Objetivo: Este artigo tem como objetivo relatar a experiência de acadêmicas de enfermagem sobre uma ação educativa que abordou os tratamentos holísticos e complementares como alternativa para os profissionais de saúde no manejo e controle da dor oncológica. Metodologia: Trata-se de um estudo descritivo, de abordagem qualitativa, do tipo relato de experiência, trazendo a vivência de acadêmicas de enfermagem de um centro universitário na região metropolitana de Belém do Pará ao realizarem uma ação educativa no local de tratamento oncológico particular no município de Belém. Relato de Experiência: Em uma roda de conversa foi elaborado uma nuvem de ideias com base no conhecimento que os mesmos possuíam, em seguida, por meio de slides foram demostrados os 4 tipos de cuidados holísticos: aromaterapia, musicoterapia, cromoterapia e acupuntura. Ao desenvolver o conhecimento, foi disponibilizado um link através do SWAY, onde os profissionais puderam consultar os materiais. Evidentemente, nesta etapa utilizamos a metodologia ativa no formato de questionário, onde várias perguntas relacionadas ao assunto foram adicionadas na plataforma Kahoot, os profissionais participantes deveriam responder as perguntas de verdadeiro ou 
falso, assim iriam acumulando pontos no jogo. Considerações Finais: A ação realizada possibilitou ampliar os conhecimentos holísticos sobre a dor oncológica dos paciente para os estagiários e profissionais da clínica. Portanto, este trabalho pôde descrever a participação e elaboração das ações educativas sobre o tema citado e concluindo que a enfermagem tem autonomia para exercer seu papel no tratamento da dor, podendo usar as práticas integrativas e complementares.

Palavras-chave: Terapias complementares; Dor do câncer; Cuidados de enfermagem.

\begin{abstract}
Objective: This article aims to report the experience of nursing students on an educational action that addressed holistic and complementary treatments as an alternative for health professionals in the management and control of cancer pain. Methodology: This is a descriptive study, with a qualitative approach, of the experience report type, bringing the experience of nursing students from a university center in the metropolitan region of Belem do Pará when carrying out an educational action in the place of private oncological treatment in the municipality of Belem. Experience Report: In a conversation circle, a cloud of ideas was created based on the knowledge they had, then, through slides, the 4 types of holistic care were demonstrated: aromatherapy, music therapy, color therapy and acupuncture. When developing knowledge, a link was made available through SWAY, where professionals could consult the materials. Evidently, at this stage we used the active methodology in the form of a questionnaire, where several questions related to the subject were added on the Kahoot platform, the participating professionals should answer the questions true or false, thus accumulating points in the game. Final Considerations: The action taken made it possible to expand holistic knowledge about cancer pain from patients to interns and clinic professionals. Therefore, this work was able to describe the participation and elaboration of educational actions on the mentioned theme and concluding that nursing has autonomy to exercise its role in pain management, being able to use integrative and complementary practices.
\end{abstract}

Keywords: Complementary therapies; Cancer pain; Nursing care.

\begin{abstract}
Resumen
Objetivo: Este artículo tiene como objetivo reportar la experiencia de estudiantes de enfermería en una acción educativa que abordó los tratamientos holísticos y complementarios como una alternativa para los profesionales de la salud en el manejo y control del dolor oncológico. Metodología: Se trata de un estudio descriptivo, con abordaje cualitativo, del tipo relato de experiencia, aportando la experiencia de estudiantes de enfermería de un centro universitario de la región metropolitana de Belém do Pará al realizar una acción educativa en el lugar de tratamiento oncológico privado. en el municipio de Belém. Reporte de experiencia: En un círculo de conversación, se creó una nube de ideas a partir de los conocimientos que tenían, luego, a través de diapositivas, se demostraron los 4 tipos de atención integral: aromaterapia, musicoterapia, cromoterapia y acupuntura. A la hora de desarrollar conocimiento, se puso a disposición un enlace a través de SWAY, donde los profesionales podían consultar los materiales. Evidentemente, en esta etapa se utilizó la metodología activa en forma de cuestionario, donde se agregaron varias preguntas relacionadas con el tema en la plataforma Kahoot, los profesionales participantes debían contestar las preguntas verdadero o falso, acumulando así puntos en el juego. Consideraciones finales: La acción realizada permitió ampliar el conocimiento holístico sobre el dolor oncológico de los pacientes a los internos y profesionales de la clínica. Por lo tanto, este trabajo logró describir la participación y elaboración de acciones educativas sobre el tema mencionado y concluir que la enfermería tiene autonomía para ejercer su rol en el manejo del dolor, pudiendo utilizar prácticas integradoras y complementarias.
\end{abstract}

Palabras clave: Terapias complementarias; Dolor de cáncer; Cuidado de enfermera.

\title{
1. Introdução
}

Sendo uma doença milenar, o câncer foi relatado pela primeira vez em papiros egípcios, manuscritos persas e indianos há cerca de trinta séculos a.C (Freitas et al., 2019). Dentre todo o avanço histórico da medicina, esta patologia já foi descrita tendo inúmeros conceitos, incidências e fatores, de acordo com a sociedade, época e religião de cada tempo histórico. No Brasil, as medidas farmacológicas para o câncer foram adotadas já no início do século XX, como medidas de controle e atendimento, os tratamentos alternativos em oncologia são em sua maioria de base popular (Freitas et al., 2019.)

Com a implantação do modelo biomédico em meados do século XX, esse conhecimento foi marginalizado por não haver embasamento científico, porém atualmente o interesse pelas terapias alternativas vêm aumentando, principalmente por não se tratar de métodos invasivos e ser acessível para todas as classes sociais. O Instituto Nacional do Câncer - INCA Brasil (2001) definiu câncer como sendo o crescimento anormal de células que invadem os tecidos e órgãos do corpo podendo espalhar-se para outras áreas e vem acompanhada de dor, e em 1979 a Organização Mundial de Saúde (OMS) definiu dor 
como uma experiência sensorial e emocional desagradável, associada a lesões reais ou potenciais. (Nascimento et al, 2017).

O sistema nervoso é responsável pela captação de estímulos. Esse sistema nervoso se constitui no Sistema Nervoso Central e Periférico que apresentam células especializadas para captar a dor. Estes receptores permitem que a sensação dolorosa seja percebida, sendo denominados, nociceptores. A dor nociceptiva pode ser causada pelo câncer se espalhando para os ossos, músculos, articulações ou algo que bloqueie um órgão ou vasos sanguíneos. Também podendo ser do tipo Dor neuropática - causada pela lesão efetiva de nervos e descrita como uma sensação pesada, de queimação, ou de dormência. (Ercolani et al 2018).

Tendo em vista a dor, essas práticas visam tratar o indivíduo de maneira integral, promovendo a saúde. Sabe-se que a dor é incapacitante, logo, muitos pacientes perdem a qualidade de vida por causa do desencadeamento de outros distúrbios, como a depressão, isolamento social, angústia e insônia. A Medicina Complementar vem ganhando força a partir da década de 70, destacando-se como alternativa na substituição de tratamentos alopáticos e procedimentos convencionais ou a fim de complementar um tratamento em andamento, visando promover a saúde e bem-estar ao paciente, diminuindo a possibilidade de efeitos colaterais e reações adversas e sendo uma opção para controle da dor do câncer (Pereira et al., 2014).

A dor oncológica é uma "dor total" tratando-se de uma síndrome, que vai além da lesão, aborda os fatores físicos, emocionais, espirituais e tem influência na expressão da queixa. Preocupados com a avaliação da dor, a OMS propôs a implementação sistemática de rotinas de avaliação, registro e controle da dor a serem incorporados, considerando-se, assim, a dor como o quinto Sinal Vital desde 2003. (Nascimento, 2017).

A terapia holística ou complementar de saúde é definida como qualquer sistema de medicina, prática ou produto que não fazem parte dos cuidados médicos convencionais. O termo "complementar" é utilizado quando a prática se associa à medicina tradicional do Ocidente, complementando o tratamento, enquanto o termo "alternativa" se aplica quando a terapia substitui completamente o tratamento convencional, Florence Nightingale foi pioneira em descrever as contribuições dos tratamentos holísticos nos cuidados de enfermagem (Sousa et al., 2021).

Levando em consideração a dor e as formas de controlar, a Aromaterapia é um exemplo muito usado para tratar problemas de saúde e proporcionar o bem-estar e a qualidade de vida, por ser baseada em substâncias não invasivas e de fácil acesso. Nela são utilizados óleos essenciais (OE) por meio de aplicação tópica, inalação ou olfato para prevenir, tratar e aliviar os sintomas a partir de derivados de plantas, obtidos da destilação ou prensagem de flores, folhas, sementes, frutos e raízes. Por sua vez, a Cromoterapia também é uma técnica milenar utilizada para implementar métodos gerais de tratamento de forma autônoma, trazendo assim uma melhoria significativa aos pacientes de forma não agressiva, em pontos específicos, para tratamento de doenças, e o equilíbrio do corpo (Pessoa et al., 2021).

Ademais, outro exemplo de suma importância é a Musicoterapia, no qual, a música sempre foi usada intuitivamente para intervir e restaurar o equilíbrio entre a mente e o corpo, refletindo o contexto cultural do paciente, aspecto importante que sempre deve ser considerado no tratamento do mesmo, especialmente quando outros comportamentos não se mostraram eficazes (Bergold \& Alvim, 2009). Além disso, ela também pode mobilizar as áreas neurais, atraídas pela estrutura do "cérebro primitivo" relacionada à motivação, prazer e emoções. (Anjos et al, 2017). Por fim, há também a acupuntura, uma técnica arcaica chinesa, que foca no diagnóstico de doenças e o progresso de cura segundo estímulo de auto cura do corpo. Esta terapia visa reajustar e redirecionar a energia estimulando pontos de acupuntura com finas agulhas de metal, lasers ou pressão para estimular os nervos periféricos e causar alterações nos neurotransmissores do sistema nervoso central, objetivando a promoção de melhora do paciente (Bousfield et al, 2021).

Por esse motivo é necessário que a equipe de saúde tenha ciência e aptidão promovendo um cuidado holístico e aprimorando os conhecimentos nas condutas terapêuticas a fim de oferecer melhor condição de vida. Um dos recursos existentes para o manejo da dor é a terapêutica medicamentosa, este recurso possibilita o controle do sintoma, entretanto, para 
alguns casos, este manejo não se mostra suficiente para a atenuação da dor ou não condiz com a escolha do paciente. Nesse contexto, observa-se a emergência de técnicas terapêuticas alternativas e complementares às convencionais para o manejo da dor de pacientes oncológicos. (Vitor et al., 2018).

Por outro lado, há evidências que a utilização de fármacos em especial os opioides por causa do grande feito analgésico por tempo prologado, pode causar diversos problemas, inclusive o vício (Nascimenton \& Sakata, 2011). Ao contrário dos efeitos adversos desse tratamento, o uso das PIC's além de não ter fator de risco no âmbito da tolerância e/ou dependência, substitui diversos recursos, inclusive o menor custo para convênios particulares e o Sistema Único de Saúde, ao ser comparado com a terapêutica medicamentosa (Neto et al., 2017).

A teoria do cuidado humano de Watson prescreve que é essencial para a sociedade atual manter os ideais de cuidado humano. A lógica de pensamento proposto por Watson pode ser desenvolvida quando é entendida as dimensões humanísticas necessárias, o pensamento também pode fazer frente ao egocentrismo e o cuidado prescritivo, sem dar voz ao sujeito que suplica pelo cuidado. Para embasar essa mudança de paradigma e romper com os padrões dominantes, pensando criticamente o cuidado, salienta-se a importância da teoria de Watson. (Riegel et al, 2018).

Essa teórica auxilia na formação e modelagem do pensamento crítico holístico dos enfermeiros, premente para o atual momento. Nesse sentido, Watson desenvolveu, em sua teoria do cuidado humano, dez fatores caritativos considerados necessidades de cuidado específicas às experiências humanas e que devem ser abordados pelos enfermeiros: sistema de valores humanísticos e altruístas, fé e esperança, sensibilidade para si e para os outros, desenvolvimento de relações de ajuda, confiança, cuidado, expressão de sentimentos e emoções positivas e negativas e o processo de cuidado criativo. (Menezes et al, 2019).

Este trabalho tem como objetivo relatar a experiência de acadêmicos do curso de enfermagem sobre uma ação educativa que abordou os tratamentos holísticos e complementares como alternativa para os profissionais de saúde no manejo e controle da dor oncológica. Portanto, baseando-se na comprovação da capacidade do enfermeiro em intervenções pautadas no tratamento holístico, evidenciando que o tratamento não invasivo pode melhorar a doença e implementar um atendimento mais humanizado, tendo eficácia nos resultados esperados de cada paciente ajudando-os a manter o equilíbrio e a melhora de forma considerável e eficaz.

\section{Metodologia}

Estudo descritivo, de abordagem qualitativa, do tipo relato de experiência, trazendo a vivência de acadêmicas de enfermagem de um centro universitário na região metropolitana de Belém do Pará ao realizarem uma ação educativa em um local de tratamento oncológico particular no município de Belém.

Foi utilizada como alicerce teórico, para desenvolver a ação educativa, a metodologia do Arco De Maguerez, que consiste em uma estratégia de ensino-aprendizagem a partir da metodologia da Problematização, o qual é composto por 5 etapas, sendo estas: a) Observando a realidade; b) Levantamento dos postos-chaves; c) Teorização; d) Hipótese de soluções; e) Aplicação à realidade (Prado et al., 2012).

Desse modo, iniciou-se o processo de elaboração do presente estudo a partir da interação e adequação das acadêmicas de enfermagem ao contexto dos atendimentos ao paciente oncológico, no contexto do manejo aos principais sinais e sintomas do câncer. A partir da interação das acadêmicas no contexto do manejo da dor do paciente oncológico, realizado por enfermeiros da unidade, elencou-se os pontos chaves do processo assistencial, o que culminou ao processo de teorização em cima dos problemas encontrados e na formulação da hipótese de solução, que resultou em uma ação educativa sobre o tratamento holístico como alternativa pelo enfermeiro no controle da dor oncológica, em novembro de 2021, com o público alvo os enfermeiros responsáveis pelos cuidados aos pacientes oncológicos da unidade, com o intuito de proporcionar aos 
participantes o conhecimento de tratamentos alternativos aos farmacológicos, de fácil manuseio e aplicação, no controle da dor desses pacientes.

Para a fundamentação teórica foram utilizados estudos relevantes que englobassem o tema pré-estabelecido, por meio de pesquisas bibliográficas realizadas nas bases de dados: Scielo, Biblioteca Virtual da Saúde (BVS) e Pubmed.

A ação educativa foi estruturada e organizada em quatro etapas, aplicadas da seguinte forma:

$1^{\mathbf{0}}$ Etapa: Foi realizado com os profissionais de enfermagem presentes uma roda de conversa, para assim elaborarmos uma "nuvem de ideias", com o conhecimento prévio que os mesmos possuíam.

$2^{\circ}$ Etapa: Aconteceu a demonstração através de slides com alguns tipos de cuidados holístico possíveis de serem aplicados naquele ambiente, sendo estes: Aromaterapia, Musicoterapia, Cromoterapia e Acupuntura.

$3^{\circ}$ Etapa: Para sintetizarmos o conhecimento, foi distribuído o link de um SWAY, criado pelas alunas, para que os profissionais pudessem consultar materiais como artigos científicos, vídeo aulas e imagens sobre os tipos de terapias holística abordadas.

$4^{\circ}$ Etapa: Por fim, nesta etapa utilizamos a metodologia ativa no formato de perguntas e respostas, onde várias perguntas relacionadas ao assunto foram adicionadas na plataforma Kahoot, os profissionais participantes deveriam responder as perguntas de verdadeiro ou falso, assim iriam acumulando pontos no jogo.

\section{Resultados e Discussão}

A ação foi realizada por seis acadêmicos do sexto semestre do curso de bacharelado em enfermagem de uma universidade privada em Belém do Pará, começou às 16:55 horas da tarde do dia 10/11/2021, conforme horário cedido pela próprio local e teve a presença dos enfermeiros e de alguns outros profissionais como nutricionistas e psicólogos. O local onde foi realizada a ação trata-se de uma clínica renomada na mesma cidade e especializada em serviços oncológicos no centro de Belém do Pará.

As ações educativas realizadas visaram generalizar e instigar os conhecimentos sobre as práticas complementares em saúde a equipe, onde foram abordados os temas de Aromaterapia, cromoterapia, musicoterapia e acupuntura, realçando a importância do domínio destes em relação aos tópicos, para melhor manejo da dor ao paciente oncológico. Houve grande participação da equipe, que apresentou diversas dúvidas e complementações sobre a temática.

No primeiro momento, foi elaborado um acolhimento aos profissionais, de forma que se sentissem a vontades no ambiente, em seguida, foi dado início a apresentação das acadêmicas, para a melhor interação com o público foi utilizada a dinâmica da "nuvem de ideias", momento em que eles colocassem suas ideias e concepções sobre o que entendem pela palavra "dor", no qual tiveram a oportunidades de expor o que pensavam, as respostas que obtivemos foi "sofrimento", "desespero", "psicológico", “medo", "agonia", “medicamentos”, "aflição", “angústia”, “infelicidade” e "tristeza”, com tudo o que foi exposto fomos debatendo sobre cada palavra dita.

No segundo momento, foi introduzido o tema "Tratamentos holísticos como alternativa para enfermeiro no controle da dor oncológica" em forma de slides, depois da explicação e conceito do holismo, foi explicado de forma sucinta sobre a teoria do cuidado humano de Jean Watson que introduziu o pensamento crítico holístico do enfermeiro. Dando-lhes um embasamento científico do assunto, e explicando-lhes sobre o conceito de cuidado e em pressupostos fenomenológicos existenciais, que trazem um olhar para além do corpo físico. Após esse momento, foi dado o início para a abordagem dos tópicos e terapias.

Ao decorrer da palestra, o primeiro tópico a ser abordado foi a aromaterapia, todos os profissionais responderam que conheciam a alternativa, porém, nenhum deles relataram fazer uso de tal. Foi exposto meios aromaterápicos para o controle da 
dor e evidenciado que os serviços de saúde atualmente têm utilizado desta alternativa para controle da dor e melhor recuperação dos pacientes hospitalizados. Florence Nightingale, pioneira no campo da enfermagem, foi uma das primeiras a introduzir os óleos essenciais no campo da enfermagem.

Segundo tópico, foi abordado a cromoterapia, seu conceito e as cores, enfatizando a cor azul, por agir como analgésico auxiliando para o controle da dor, também esclarecido sobre a lei federal $n^{\circ} 7.498$, quem em seu art. 11, afirma que a cromoterapia está nas atribuições do enfermeiro legalizadas pelo conselho federal de enfermagem (COFEN), houve pouca interação em relação a esse tópico, entretanto ele foi muito bem explicado e entendido pelo público.

Em seguida, o terceiro tópico apresentado foi sobre Acupuntura, foi feito uma pergunta a eles se tinham visto ou já tinham feito alguma sessão e se poderiam contar para os que estavam presentes, apenas um profissional contou como havia sido sua experiência e relatou que usou agulhas na região da testa para elucidar a cefaleia, além do mais, ressaltou que teve um resultado positivo do alívio da dor. Logo mais, foi explicado sobre o conceito, aplicações, especialidade da enfermagem, as indicações e contraindicações, também foi apresentado a resolução do COFEN que reconhece a acupuntura como uma especialidade e/ou qualificação do profissional de enfermagem, foi nesse momento em que surgiram dúvidas do público e no mesmo momento foram esclarecidas.

E por fim, a Musicoterapia sendo enfatizada como um dos temas em que mais houve interações, pelo simples fato da música estar presentes em muitos ambientes. A música foi apontada como instrumento já conhecido e utilizado para a mudança de comportamentos, sentimentos ou fatores fisiológicos por décadas, incluindo a enfermeira Florence Nightingale, que evidenciava o poder da música no ambiente hospitalar na recuperação dos doentes. Diante dos aspectos físicos e emocionais, a interação musical é um recurso comunicacional que coopera com a diminuição de distúrbios psíquicos, emoções negativas e estilos de comportamento, beneficiando o âmbito terapêutico ao promover a mobilização de ondas sonoras, em frequência e amplitudes adequadas, que vão estimular diversas sensações, agindo principalmente nos sistemas nervoso, endócrino e cardiovascular, motivando cada vez mais os pacientes a encararem a vida com mais energia, motivação e determinação.

De forma complementar, foi utilizado e disponibilizado aos profissionais uma das tecnologias chamada sway, que facilita a criação e compartilhamento de conteúdo para que os mesmos pudessem obter mais informações sobre o tema que era condizente ao do slide e consultar o material sempre que quiserem. Em seguida, como metodologia ativa, os profissionais foram instruídos pelas acadêmicas para acessar o site kahoot, uma tecnologia educacional de testes, que continham perguntas sobre o tema abordado e para que pudéssemos analisar seus conhecimentos. Eles acessaram a gamificação e a equipe observou uma interação positiva de todos eles, momento de risadas e competições entre eles. Ao término da dinâmica, eles elogiaram e gostaram da metodologia ativa, tanto que, iriam aderir a ideia na clínica com os estagiários.

Diante disso, foi evidenciado na literatura estudos que abordam o tema. De acordo com Aquino (2021). As práticas alternativas utilizadas por pacientes oncológicos têm sido descritas satisfatoriamente por proporcionarem: "sensações de bemestar e saúde". Esse benefício vem devido a prática ser abrangente, permitindo a cada paciente escolher qual a forma que mais se adapta a sua crença e religiosidade, gerando uma prática de concepções expandidas no cuidado e autocuidado, permitindo ao próprio paciente participar de sua cura gerando a superação dela.

Esse pensamento também se firma quanto a teoria do cuidado holístico de Jean Watson, abordado anteriormente neste estudo, prescreve que é essencial para a sociedade atual manter os ideais de cuidado humanístico. Sendo relevante pois há uma proliferação de tratamentos e técnicas que comumente desconsideram os aspectos humanos que envolvem o cuidado autêntico (Riegel et al, 2018). Nessa abordagem holística, acredita-se que os profissionais de saúde devem enxergar a doença de forma holística, mostrando ao paciente os múltiplos fatores que levaram à disfunção e ensinando-lhe sobre a natureza e o significado de sua doença. É fundamental que os profissionais mostrem ao paciente como ele pode mudar o estilo de vida que o conduziu à enfermidade. Tendo como objetivo restaurar a saúde integral, e não simplesmente combater a doença eliminando seus sinais e 
sintomas. (Riegel et al, 2018).

Segundo Nascimento (2017) O papel da enfermagem é indispensável para um tratamento completo e humanizado e também incluindo os tratamentos paliativos que requer muitos dos nossos olhares holísticos na hora de se posicionar com os pacientes e os seus familiares. Neste sentido, nas últimas décadas o conhecimento, o conceito e as intervenções terapêuticas para a dor crônica do paciente oncológico tiveram uma grande evolução, porém, a capacitação do enfermeiro e equipe ainda é inadequada, ao enfermeiro cabe aperfeiçoar este cuidado, sendo um bom avaliador dos sintomas e suas intensidades, atuando preventivamente quanto às complicações indesejáveis, realizando um manejo adequado de lesões e limitações impostas pelo agravo da doença oncológica avançada.

Ferreira (2021) descreve que é possível observar entre os estudos a carência por parte do curto tempo de acompanhamento e duração das intervenç̃es, tamanho limitado das amostras e a falta de padronização entre os protocolos de tratamento com as terapias complementares para a possível replicação em outros pacientes. Observou-se que a dor oncológica ultrapassa o limiar da dimensão física, pois, ao penetrar no contexto de vida da pessoa doente, passa a se sentir limitada e dilacera, além de modificar o cotidiano. Ademais, altera os hábitos no campo social e de lazer, assim como as necessidades básicas, desde a alimentação ao padrão de sono, sendo também expressa pelas muitas dificuldades para manter as atividades habituais.

Diante dessa situação, é importante avaliar e orientar os profissionais de saúde com conhecimentos sobre a prática integral e complementar (PIC) para determinar as principais modalidades de tratamento utilizadas pela equipe assistencial, pois além da terapia medicamentosa, pode ser utilizada a terapia não medicamentosa como alternativa ao alívio da dor. No entanto, seguindo a tendência do modelo biomédico, a terapia medicamentosa ainda domina (Azevedo et al, 2019). A dificuldade do cuidado integral está no cuidado às pessoas, desde o cuidado propriamente dito ao cuidado às pessoas, ressaltando-se a importância da sistematização desse tipo de cuidado, visando à avaliação do enfermeiro, avaliação de parâmetros, controle e registro da dor, para promover a Integralidade da assistência de enfermagem no cuidado aos pacientes que estão recebendo tratamento para a dor (Silva et al, 2021).

Portanto, a importância do enfermeiro no papel da educação, por meio do diálogo com os pacientes, orientação e esclarecimento de dúvidas sobre o diagnóstico e tratamento, bem como a atuação na escuta de suas decisões, tornou-se um fator determinante na qualidade da assistência ao paciente oncológico. A ação educativa é um dos instrumentos que o enfermeiro deve aprender em sua jornada acadêmica. De acordo com Figueiredo (2020) A formação inicial em enfermagem tem um papel essencial no desenvolvimento de competências para ação educativa. As Diretrizes Curriculares Nacionais (DCNs), em vigor desde 2001, reafirmam a necessidade e o dever das Instituições de Ensino Superior de formar profissionais de saúde para o SUS, com a finalidade de adequar a formação em saúde às necessidades de saúde da população brasileira.

As práticas educativas realizadas por enfermeiros no cotidiano da assistência têm enfatizado a transmissão de informações e a mudança de comportamentos dos indivíduos. Vincula-se a um modelo de atenção à saúde voltado para a doença, com ênfase no conhecimento técnico-científico especializado. Porém, as PIC ainda não têm espaço e repercussões considerável, logo, é imprescindível que a enfermagem continue resgatando esse equilíbrio de ciência e tradição, não só na atenção primárias, mas nos outros dois níveis de atenção. É importante transmitir os princípios de integridade e autonomia, que é a capacidade do ser humano de decidir algo com base em seus próprios valores, expectativas, necessidades, prioridades e crenças (Azevedo et al., 2019).

\section{Considerações Finais}

Muitas patologias e sintomas têm origem energética, demonstrando que o estado de saúde se relaciona diretamente com o ambiente. Desta forma, com a inclusão dos profissionais e o maior conhecimento sobre o tratamento holístico é possível 
tratar os pacientes com outra terapêutica, não sendo a farmacológica como principal escolha. Ademais, a atividade realizada possibilitou ampliar o conhecimento dos estagiários e profissionais da clínica sobre as práticas holística e como pode ser utilizada nos pacientes com dor oncológica. Portanto, é notório que o uso dessa terapêutica ainda está na fase de implantação nas clínicas e no Sistema Único de Saúde. Sendo assim, faz-se necessário a capacitação de mais profissionais para esta área de atuação. Portanto, este trabalho pode descrever a participação e elaboração das ações educativas sobre o tema citado e concluindo que a enfermagem tem autonomia para exercer seu papel no tratamento da dor, podendo usar as práticas integrativas e complementares.

\section{Agradecimentos}

Agradecemos a todos que direta e indiretamente contribuíram para a realização e sucesso do artigo.

\section{Referências}

Anjos, A. G., Montanhaur, C. D., Campos, E. B. V., Piovezan, J. S. M \& Neme, C. M. B. (2017). Musicoterapia como estratégia de intervenção psicológica com crianças: uma revisão da literatura. Revista Interinstitucional de Psicologia, 10(2), 228-238.

Azevedo, C., Moura, C. C., Corrêa, H. P., Mata, L. R. F., Chaves, E. C. L \& Chianca, T. C. M. (2019). Práticas integrativas e complementares no âmbito da enfermagem: aspectos legais e panorama acadêmico-assistencial. Escola Anna Nery, 23(2), 20211-110.

Bergold, L. B \& Alvim, N. A. T. (2009). A música terapêutica como uma tecnologia aplicada ao cuidado e ao ensino de enfermagem. Revista escola Anna Nery, 13(3), 537-542.

Bousfield, A. P. S., Padilha, M. I., Bellaguarda, M. L. D. R., \& Costa, R. (2021). Processo de Enfermagem como potencializador da prática da acupunturaa. Escola Anna Nery, 25.

Contatore, O. A., Tesser, C. D. \& Barros, N. F. (2018). Medicina chinesa/acupuntura: apontamentos históricos sobre a colonização de um saber. História, Ciências, Saúde-Manguinhos, 25, 841-858.

Ercolani, D., Hopf, L. B. S., \& Schwan, L. (2018). Dor crônica oncológica: avaliação e manejo. Revista Acta Médica, 39(2), 151-162.

Ferreira, P. M., Souza, T. C., Freitas, P. S., Bressan, V. R., Silva, L. J. A., \& Terra, F. S. (2021). Uso das práticas integrativas e complementares pela enfermagem em pessoas com câncer: revisão integrativa. Brazilian Journal of Health Review, 4(1), 1841-1858.

Figueiredo, A. M., Reis, D. P., Pimenta, A.C.A., Santos L. J. C., Frazão, J.C., Silva, M. C. R. Cunha, F. F., Silva F. A., Spindola, P. R. N., Santos B. N., Azevedo, B. A. R., Lopes. M. M. B., Vasconcelos, P.L.S. Paixão, A. R. T, \& Castro H. C. (2020). Percepção de acadêmicos de Enfermagem sobre educação em saúde na perspectiva da qualificação do cuidado. Revista Eletrônica Acervo Saúde, 12(1), 19-64.

Freitas, W. M. L., Nunes, M. S., Rodrigues, P. K. B., Silva, L. F., Martins, T. M., \& Silveira, M. F. (2019). História familiar de câncer, perfil sociodemográfico e estilo de vida da população assistida em um programa de prevenção ao câncer. Unimontes Científica, 93-100.

Menezes, T. A., Santana, I. G., \& Rodrigues, I. D. (2019). Teoria do cuidado transpessoal e suas contribuições no âmbito da assistência de Enfermagem: uma revisão sistemática. In: Congresso Internacional de Enfermagem.

Nascimento, D. C. H., \& Sakata, R. K. (2011). Dependência de opióide em pacientes com dor crônica. Revista dor, 12,160-165.

Nascimento, J. C. C. (2017). Avaliação da dor em paciente com câncer em cuidados paliativos à luz da literatura. SAÚDE \& $C I \hat{E} N C I A E M A C ̧ A \tilde{O}, 3(1), 11-26$.

Neto, M. R. C., Aquino, C. M. F., Sousa, I. M. C., \& Assunção, M. C. T. (2017). Investigação de custos em pics: estudo de caso de uma unidade especializada na rede de atenção à saúde, recife/pe. Anais CONGREPICS... Realize Editora.

Pereira, R. D. M., Silva, W. W. O., Ramos, J. C., Alvim, N. A.T., Pereira, C. D., \& Rocha T. R. (2014). Praticas integrativas e Complementares de saúde: Revisão integrativa sobre medidas não farmacológicas à dor oncológica. Revista de Enfermagem da UFPE on line, 9(2), 710-717.

Pessoa, D. L., Santos, B. O., Abreu, C. B. R., Mendes, K. F. C., Hora, M. C. C., Cantanhede, M. C., Salles, R. R., Lima, S. F., Carvalho, M. S. C., \& Amorim, C. E. N. (2021) O uso da aromaterapia na prática clínica e interprofissional. Research, Society and Development, 10(3), e46410313621-e46410313621.

Riegel, F., Crossetti, M. G. O., \& Siqueira, D. S. (2018). Contribuições da teoria de Jean Watson ao pensamento crítico holístico do enfermeiro. Revista Brasileira de Enfermagem, 71, 2072-2076.

Prado, M. L., Velho, M. B., Espíndola, D. S., Sobrinho, S. H., \& Backes, V. M. S. (2012). Arco de Charles Maguerez: refletindo estratégias de metodologia ativa na formação de profissionais de saúde. Escola Anna Nery, 16 (1), 172-177.

Silva, E. R., Felix, J. M \& De Freitas, M. R. I. (2021). O uso da musicoterapia como prática integrativa e complementar do enfermeiro no ambiente hospitalar. Brazilian Journal of Development, 7(10), 95228-95236. 
Research, Society and Development, v. 10, n. 16, e129101623418, 2021

(CC BY 4.0) | ISSN 2525-3409 | DOI: http://dx.doi.org/10.33448/rsd-v10i16.23418

Sousa, L. A., Salim, N. R., Fumincelli, L., \& Texeira, I. M. C. (2021). Complementary therapies in education, community extension and research in nursing. Revista Brasileira de Enfermagem. 74(2), 01-08.

Tavares A. T. A, Anjos T. S., Oliveira S. S., Andrade E. A., Silva G. K. R., Silva V. A. A. \& Ferreira L. L. (2021). Manejo da dor oncológica pela equipe de enfermagem. Research, Society and Development, 10(11), e4721011198544.

Vitor, A. H., Barros, D. H. S., \& Barbosa, R. M. (2020) Terapias alternativas para o alívio da dor em pacientes oncológicos. 